\title{
Análisis cladístico de la familia Agavaceae
}

\author{
LUIS HERNÁNDEZ SANDOVAL
}

Escuela de Biología. Universidad Autónoma de Querétaro. Centro Universitario, Querétaro 76013, Qro. México.

\begin{abstract}
Resumen. Se presenta un análisis cladístico con base en caracteres morfológicos de 20 taxa pertenecientes o asociados a la familia Agavaceae. Para encontrar caracteres homólogos e independientes entre sí, se analizó la morfología de cada taxón bajo los criterios de que: a. las plantas tienen una arquitectura modular; b. la morfología de las plantas evoluciona mediante cambios ontogenéticos; c. los caracteres evolucionan a través de cambios secuenciales. Esto permitió que se propusieran hipótesis sobre evolución de los caracteres, a probarse conjuntamente con métodos comparativos y cladísticos. Al optimizar los caracteres con el método de parsimonia de Wagner, mediante el programa PAUP 3.1.1, proponiendo los géneros Chlorogalum y Camassia (Hyacinthaceae) y Chlorophytum y Eremocrinum (Anthericaceae) como grupo externo múltiple, se obtuvieron dos árboles filogenéticos con 163 pasos de longitud y un Índice de Consistencia de 0.509. En el árbol de consenso estricto se puede observar que el grupo hermano de Agavaceae está formado por géneros de la familia Funkiaceae (Hesperocallis, Hosta). Chlorophytum y Camassia, considerados en estudios basados en biología molecular como cercanos a la familia, resultaron más lejanos en este análisis. Dentro de las Agavaceae sensu stricto se separaron los grupos reconocidos como subfamilias Yuccoideae y Agavoidae. El primero está estrechamente relacionado con el género sudamericano Excremis (Funkiaceae), del cual aún no se tiene suficiente información morfológica como para asegurar su inclusión en Agavaceae. En la base de las Agaveae resultó Hesperaloë, seguido de los taxa hermanos Beschorneria y Furcraea, después Agave asociado a Manfreda y géneros afines y finalmente, como grupo más derivado a los géneros Polianthes y Pseudobravoa.
\end{abstract}

Palabras clave: Agavaceae, cladística, homología, morfología.

Abstract. A morphological based cladistical analysis was undertaken, using 20 taxa from or associated to the family Agavaceae. To assess homologous and independent characters, the morphology of each taxa was analyzed under the next criteria: a. plants have modular construction; b. plant morphology evolves through ontogenetic changes; $c$. morphological characters evolve through secuential modifications. Hypotheses on character evolution were proposed and tested with comparative and cladistical methods. The morphological characters were optimized with the Wagner parsimony method using PAUP 3.1.1, considering the genera Chlorogalum and Camassia (Hyacinthaceae), and Chlorophytum and Eremocrinum (Anthericaceae) as multiple outgroups. Two equal most parsimony trees were obtained, 163 steps long with a Consistency Index of 0.509. From the strict consensus tree, genera of Funkiaceae (Hesperocallis, Hosta) resulted as sister taxa of Agavaceae. Chlorophytum and Camassia, considered in molecular biology studies as closely related to Agavaceae, resulted further down. Within the Agavaceae sensu stricto, the previously re ognized groups Yuccoideae and Agavoideae as subfamilies, appeared well delimited. The first group is associated witil the South American genus Excremis (Funkiaceae) The morphological data from Excremis is not enough as to consider it within Agavaceae. At the base of the Agaveae clade, Hesperaloë resulted as the sister taxon of the group, followed by the sister taxa Beschorneria and Furcraea. Finally, the group considered by authors as the tribe Agaveae formed a monophyletic clade with Agave at the base associated to Manfreda and relatives, and the sister group Polianthes and Pseudobravoa as the most derived taxa.

Key words: Agavaceae, cladistics, homology, morphology.

\section{INTRODUCCIÓN}

La familia Agavaceae sensu Dahlgren et al. (1985) pertenece al orden Asparagales; contiene $c a .300$ especies en diez géneros, agrupados en las subfamilias Yuccoideae y Agavoideae. Todas las especies se distribuyen en el continente americano.

A través de la historia, la clasificación de los géneros pertenecientes a esta familia ha variado de acuerdo con los enfoques de diversos autores (Kunth, 1850; Baker, 1871, 1873, 1881; Rose, 1899; Lotsy, 1911; Pax y Hoffman ,1930; Hutchinson, 1934, 1959; Traub, 1953; Takhtajan, 1980; Cronquist ,1981; Schulze, 1983; Dahlgren et al., 1985). Estas variantes se han debido principalmente a la falta de entendimiento en la evolución de la morfología, en casos como hábito, crecimiento secundario y posición del ovario en las flores. Con respecto a los caracteres citológicos, en las últimas propuestas de clasificación se consideró a Hosta como uno de los taxa estrechamente relacionados con las Agavaceae por su gran similitud (Traub, 1953; Takhtajan 1980).

Recientemente, con métodos de parsimonia, se han realizado estudios que han contribuido al esclarecimiento de las relaciones filogenéticas de la familia (Hernándezy Simpson en prep.). Hernández (1993) c cun base en un análisis sobre evolución de la morfología de las Asparagales americanas encontró que Hesperocallis es el género hermano de Agavaceae. Duvall et al. (1993) y Eguiarte et al. (1994) con informacir de secuencias de $r b c L$, proponen a Chlorophytum como un pariente cercano de Agavaceae. 
Bogler y Simpson (1995), en su estudio sobre Agavaceae basado en sitios de restricción de ADN de cloroplasto, encuentran que Hosta (Funkiaceae) es el género hermano de la familia. Finalmente, utilizando secuencias del gen ITS (Bogler en prep.) coincide con la información de Chupov y Kuntjavina (1981), basada en serología, de que Camassia es un género muy relacionado con $Y u c c a$.

El objetivo de este trabajo es inferir la filogenia de la familia Agavaceae, utilizando caracteres morfológicos y, toma como grupos externos a aquellos taxa que han sido considerados como sus parientes cercanos.

\section{MÉTODOS}

Se realizó un análisis cladístico de 20 taxa pertenecientes o afines a la familia Agavaceae sensu Dahlgren et al. (1985), utilizando 49 caracteres morfológicos (Cuadros 1 y 2). Los caracteres se obtuvieron de plantas vivas, ejemplares de herbario (MEXU, TEX, BM) y de la literatura. Las referencias utilizadas fueron las siguientes:
Tratamientos generales (taxa supragenéricos o información parcial de géneros). Baker (1871, 1873, 1881), Dahlgren y Clifford (1982), Dahlgren et al . (1985), Pax y Hoffman (1930), Rose (1899, 1903), Schulze (1983), VerhoekWilliams (1975).

Caracteres (taxa supragenéricos o información parcial de géneros). Hábito y arquitectura vegetal: Hallè et al. (1978), Tomlison (1984). Haces vasculares (vasos): Cheadle (1969), Cheadle y Kosakai (1971). Hojas: Blunden et al. (1973), McLendon (1908). Estomas: Álvarez (1985, 1990). Plántulas: Boyd (1932). Inflorescencias: Álvarez (1986), Troll (1964). Nectarios florales: Daumann (1970). Embriología: Cave (1948, 1974), Wunderlich (1950). Palinología: Álvarez y Köhler (1987), Ojeda et al. (1984), Ojeda (1988). Semillas: Huber (1969). Citología: Cave (1964), GómezPompa et al. (1971), McKelvey y Sax (1933), Satô (1935), Sen (1975), Whitaker $\{1934)$.

Taxa. Agave: Gentry (1982). Beschorneria: GarcíaMendoza (1987). Camassia: Gould (1942), Leffigwell (1930). Polianthes: Joshi y Pantulu (1941). Yucca: Arnott (1962),

CUADRO 1. Lista de caracteres y sus estados

Vegetativos

* 1-2. Arquitectura (hábito):

00 monocárpica aérea - 10 policárpica aérea <

11 subterránea vertical

20 subterránea horizontal

3. Crecimiento secundario: 0 ausente, 1 presente

4. Raíces: 0 fibrosas, 1 carnosas

5. Raíces contráctiles: 0 ausentes, 1 presentes

6. Primera hoja (bráctea) del tallo: 0 decidua, 1 persistente

7. Superficie de hojas: 0 acanaladas o con costilla, 1 lisa

*8. Márgenes de hoja: 0 espinosas - 1 microdenticuladas - 2 enteras - 3 fibrosas

*9. Apice de hoja: 0 espinoso - 1 decurrente - 2 entero

10. Forma de células epidérmicas: 0 isodiamétricas - 1 largamente hexagonales -2 rectangulares

11. Paredes celulares de epidermis: 0 sinuosas, 1 rectas, 2 con engrosamientos

12. Tipo de estomas: 0 anomocíticos, 1 paracíticos, 2 tetracíticos

13. Tipo de germinación (Dahlgren et al., 1985): 0 tipo A, 1 tipo B, 2 tipo C

14. Filotaxia en plántulas: 0 dística, 1 espiral

\section{Reproductivos}

*15. Entrenudos de zona estéril, ZE (Troll, 1964):

() no reducidos - 1 reducidos basalmente - 2 reducidos totalmente (escapos desnudos) (inflorescencias sésiles)

16. Hojas de ZE: 0 foliosas, 1 brácteas

17. Bases de hojas de ZE: 0 no envainantes, 1 envainantes

18. Textura de hojas de ZE: 0 coriáceas, 1 suculentas

19. Hojas de zona de floración, sustentando paraclados (Troll, 1964): 0 foliosas, 1 brácteas

*20. Patrón de la última unidad de inflorescencia:

0 cincino - 1 ripidio - 2 bifloral - 3 secundariamente solitaria 
21. Forma de flores: 0 campanulada, 1 funelforme, 2 tubular

22. Simetría floral: 0 actinomórfica, 1 zigomórfica

23. Tipo de flores: 0 hipóginas, 1 epíginas

24. Segmentos del perianto: 0 libres - 1 fusionados en la base - 2 fusionados

$* 25$ Inserción de filamentos: 0 basal - 1 perigonal - 2 arriba de ovario - 3 en boca de tubo

26. Extensión de filamentos (posición de anteras): 0 insertos, 1 exertos

27. Epidermis de filamentos: 0 glabra, 1 con tricomas

28. Bases de filamentos: 0 cilíndricos, 1 planos

*29. Apices de filamentos: 0 planos, 1 filiformes, 2 glabros

30 Inserción de anteras: 0 basal, 1 dorsal

*31. Arreglo de tecas: 0 sagitadas - 1 sagitadas en la base - epipeltadas

*32. Capas del endotecio: 0 una - 1 dos - 2 tres - 3 cuatro a cinco

33. Nectarios septales en ovario: 0 distal - 1 medial -2 basal - 3 ausente

*34. Lóbulos del estigma: 0 truncados- 1 lineares -2 deltoideos -3 redondeados

35. Placentación axilar de óvulos: 0 a lo largo del eje, 1 central

36 Células parietales de óvulos: 0 ausentes, 1 presentes

37. Tipo de formación del saco embrionario: 0 Drusa, 1 Polygonatum

38. Granos de polen: 0 mónadas, 1 tétradas

39. Forma de granos de polen: 0 ahusada, 1 ovoide, 2 elipsoide

40. Aperturas de granos de polen: 0 monocolpadas, 1 bicolpadas, 2 operculares

41. Ornamentación de exina: 0 microrreticulada, 1 reticulada, 2 reticulada-heterobrocada

42. Márgenes de colpos: 0 no diferenciados 1 diferenciados

43. Tipo de frutos: 0 cápsula septicida - 1 indehiscente - 2 cápsula loculicida

*44. Forma de semillas: 0 planas lateralmente - 1 anguladas - 2 planas dorsiventralmente

45. Superficie de testa: 0 lisa, 1 escabrosa

46. Endospermo: 0 ruminado, 1 no ruminado

47. Embrión: 0 erecto, 1 curvo

*48-49. Números cromosómicos:

$00(24=6$ grandes +18 chicos $)-10(6-7)-20(14-15)<$

$30(30=6 \mathrm{~g}+24 \mathrm{ch})$

$31(30=5 \mathrm{~g}+25 \mathrm{ch})$

* Caracteres ordenados en el segundo análisis.

Cuadro 2. Matriz de datos

Agave

Agave (Litaea)

Beschorneria

Chrologalum

Camassia

Clorophytum

Erecroninum

Excremis

Furcraea

Hesperaloë

Hesperocalis

Hosta

Leucocrinum

Manfreda

Polianthes

Prochnyanthes

Pseudobravoa

Yucca

Clistoyucca

Hesperoyucca
0000000001111111111222222222233333333334444444444 1234567890123456789012345678901234567890123456789
1110001000220100011120222101112303020020210200131 1110000000220101001220222101112203020020210200131 $1110101110220101001020222011211112020122210200 ? 31$ $2000110111101 ? 11122300000001112 ? ? 2110010110110120$ $200111022111 ? ? 111010000000001122 ? 1010010200100020$ 2001010111102001101100000000101022100010200110010 200111021110 ?? 01101100000001001 ?? 21200101001111 ?? 100100011110 ? $001001000011011200 ? ? 00$ ? 0000 ??211???? 0010000000220101011000121001211130020122210200131 $11100003022 ? 0101001120000001011123020010210210031$ $110100021100 ? ? 01101301011001102 ? 03000020210210000$ $1101000210001000101310011000111 ? 03021020210010 ? 30$ $110101012100 ? 120100110222001001 ? ? 31200201101$ ? 020 1111100010220101001320222101112102020020210200131 1111100121120101001220222001112101020021110200131 1111100120120101001211222001112101020021210200131 1111100121120101001320223001112101020021110200131 $1011000302 ? 10101001000010010201120020000001211031$ 1011001101020101001000010010201120020000001211031 1011000101010101001000000010201123020000002200031 
Blunden y Binns (1970), Indamar et al. (1977), McKelvey (1938, 1947), Webber (1953).

Para obtener los árboles filogenéticos se realizó un análisis de caracteres, mediante:

1. Pruebas de hipótesis para caracteres homólogos e independientes entre sí, basadas en Patterson (1988) y Sattler (1994), utilizando los criterios de similitud, conjunción (dos caracteres considerados homólogos no pueden estar en el mismo organismo) y congruencia (distribución de los caracteres en la filogenia).

2. Propuestas de árboles de estados de los 49 caracteres, considerándolos como caracteres transmodales (aquellos para los que se invocan teorías evolutivas específicas) y morfoclinas (Mikevich y Weeler, 1990). Los caracteres en los árboles de los estados ramificados se codificaron utilizando el método de codificación ordinal propuesto por Mikevich y Weeler (1990), donde se dan números secuenciales a los caracteres que conforman la rama más larga del árbol de estados y en las ramas más cortas se mantiene el número que le corresponde al nudo que las origina. Después, en estas ramas cortas, se inicia otra secuencia a partir del cero, y a los caracteres de la rama larga que no están conectados secuencialmente con esta rama corta, se les mantiene el número de la intersección. Esto da la apariencia de codificación binaria; sin embargo, ésta representa polimorfismos y la codificación ordinal no. El resto de caracteres mantendrá el número que le corresponda al nudo que los origina (Cuadro 1, carácter 1).

3. Pruebas de hipótesis sobre evolución de caracteres para inferir la filogenia del grupo, optimizándolos con métodos de parsimonia (Mikevich y Weeler, 1990).

En los dos primeros casos, se utilizaron los criterios de que: a. las plantas tienen una arquitectura modular; b. la morfología vegetal evoluciona mediante cambios ontogenéticos; c. Los caracteres evolucionan a través de cambios secuenciales (Hernández, 1993). Para el tercer punto se utilizó el programa PAUP 3.1 1 (Swofford, 1990), polarizando los caracteres con la opción de enraizamiento por grupos externos múltiples: Camassia y Chlorogalum (Hyacinthaceae) y Chlorophytum y Eremocrinum (Anthericaceae). Se realizaron dos análisis, uno con los estados de los 49 caracteres desordenados y otro adicional considerando sólo 12 caracteres ordenados del total (Slowinski, 1993) (Cuadro 1). Los análisis se realizaron con la búsqueda heurística al azar Mulpars de Wagner (100 réplicas por intercambio de ramas, «branch-swapping»), optimizada por ACCTRAN. El análisis de Bootstrap se hizo considerando 100 réplicas con la opción de adiciones secuenciales, «stepwise addition» al azar.

\section{RESULTADOS}

Se probó la estructura de la matriz de datos con la opción de árboles al azar (Hillis y Huelsenbeck, 1992), estimando el número posible de árboles por cada longitud, obteniendo valores globales satisfactorios de $\mathrm{gl}=-0.535$. El análisis con caracteres desordenados produjo dos árboles igualmente más parsimoniosos con una longitud de 163 pasos, un índice de consistencia $\mathrm{IC}=0.509$ y un índice de retención $\mathrm{IR}=$ 0.665 . En el análisis con 12 caracteres ordenados, los dos árboles tuvieron una longitud de 179 pasos, un IC $=0.464$ y un $\mathrm{IR}=0.643$ (Figs. 1, 2, 3).

En cada árbol de consenso estricto de los dos análisis se obtuvo una topología similar. En los dos se puede observar que el grupo hermano de Agavaceae está formado por géneros de la familia Funkiaceae (Hesperocallis, Hosta). Los géneros Chlorophytum y Camassia, considerados en estudios basados en biología molecular como grupos hermanos de la familia, resultaron más lejanos en este análisis. Dentro de las Agavaceae sensu stricto se separaron los grupos reconocidos como subfamilias Yuccoideae y Agavoideae. La primera está estrechamente relacionada con el género sudamericano Excremis (considerado anteriormente en Funkiaceae). En la base de las Agaveae resultó Hesperaloë, seguido de Beschorneria y Furcraea como taxa hermanos, después Agave asociado a Manfreda y géneros afines y finalmente, como grupo más derivado a los géneros Polianthes y Pseudobravoa.

Entre los dos análisis se encontraron diferencias en los clados de la subfamilia Yuccoideae y de los géneros relacionados con Agave. En la optimización con caracteres desordenados, el árbol de consenso estricto (Fig. 2) resultó con Excremis y Hesperoyucca formando una politomía con la rama que contiene a Yucca y Clistoyucca. El grupo de Agave y géneros afines resultó monofilético.

En el análisis con 12 caracteres ordenados (Fig. 3), el clado de las Yuccoideae se expresó con Hesperoyucca en la base y el resto de taxa sin resolución. La rama que contiene a Agave no se pudo resolver con Agave subgénero Littaea ni con el grupo de Manfreda y parientes.

En el análisis de caracteres desordenados se obtuvieron los valores sobre índices de consistencia de cada carácter para evaluar su congruencia con las hipótesis sobre evolución de caracteres propuestos (Cuadro 3). Por lo tanto, como prueba de hipótesis en este estudio sólo se consideran congruentes con la filogenia, aquellos caracteres con I.C.: 1.0, $0.750,0.667$ (mayores a 0.5 ).

Los caracteres con IC $=1.0$ fueron: hábito (en parte, ya que por la codificación ordinal estuvo compuesto por otra parte con IC $=0.333$ ), primera hoja (bráctea) del tallo, tipo de germinación, entrenudos de la zona estéril (ZE), bases de las hojas de la zona estéril, las hojas de la zona de floración (ZF) sustentando paraclados, tipos de formación del saco embrionario, granos de polen (mónadas o tétradas) siete aperturas de los granos de polen, tipos de frutos, números cromosómicos. Los caracteres con IC $=0.750$ fueron capas del endotecio y nectarios septales en ovario. Los caracteres con IC $=0.667$ fueron textura de hojas de la zona estéril, tipos de flores, células parietales de óvulos, forma de granos de polen y forma de semillas. Del total de caracteres (17) 


\section{Arbol 1}

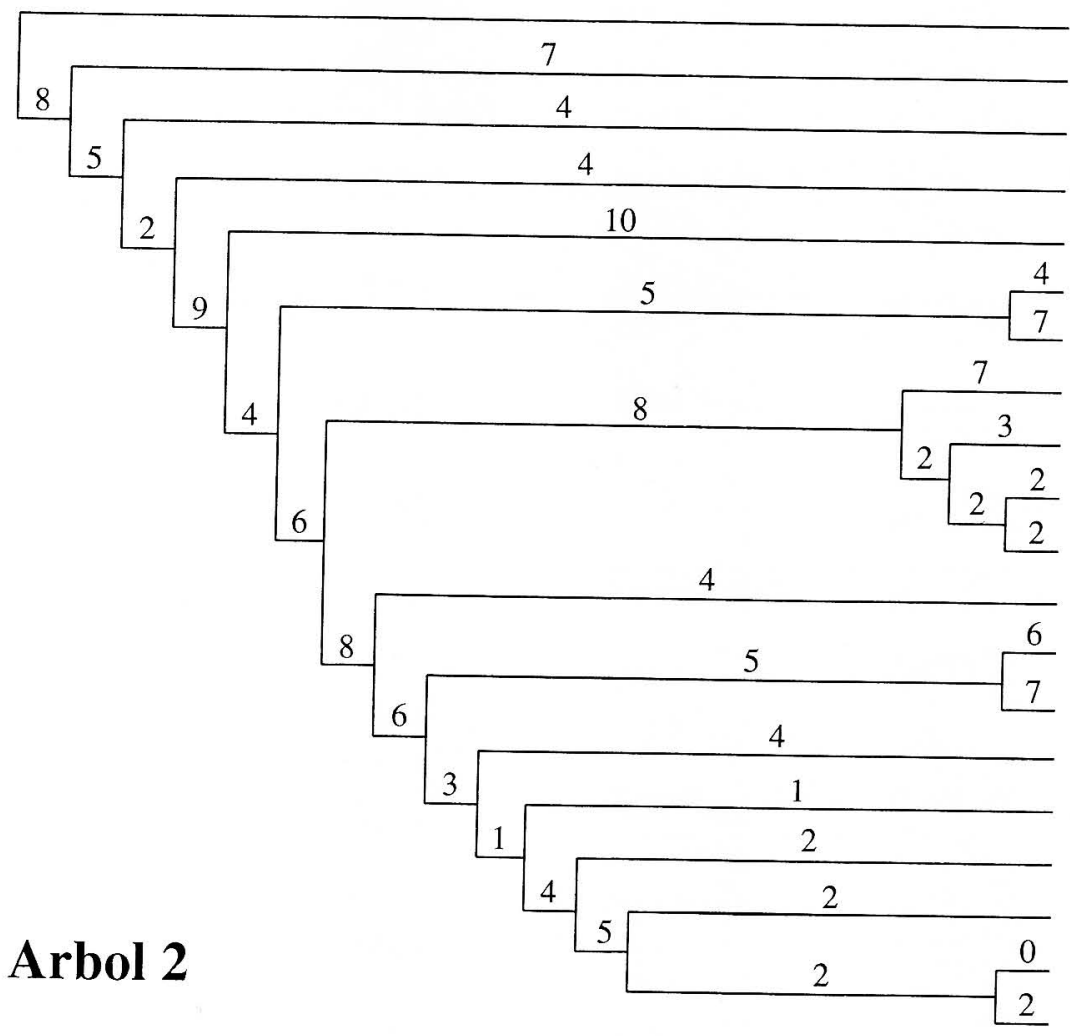

Chlorogalum

Camassia

Eremocrinum

Chlorophytum

Leucocrinum

Hesperocallis

Hosta

Excremis

Hesperoyucca

Yucca

Clistoyucca

Hesperaloë

Beschorneria

Furcraea

Agave

Agave (Litt.)

Manfreda

Prochnyanthes

Polianthes

Pseudobravoa

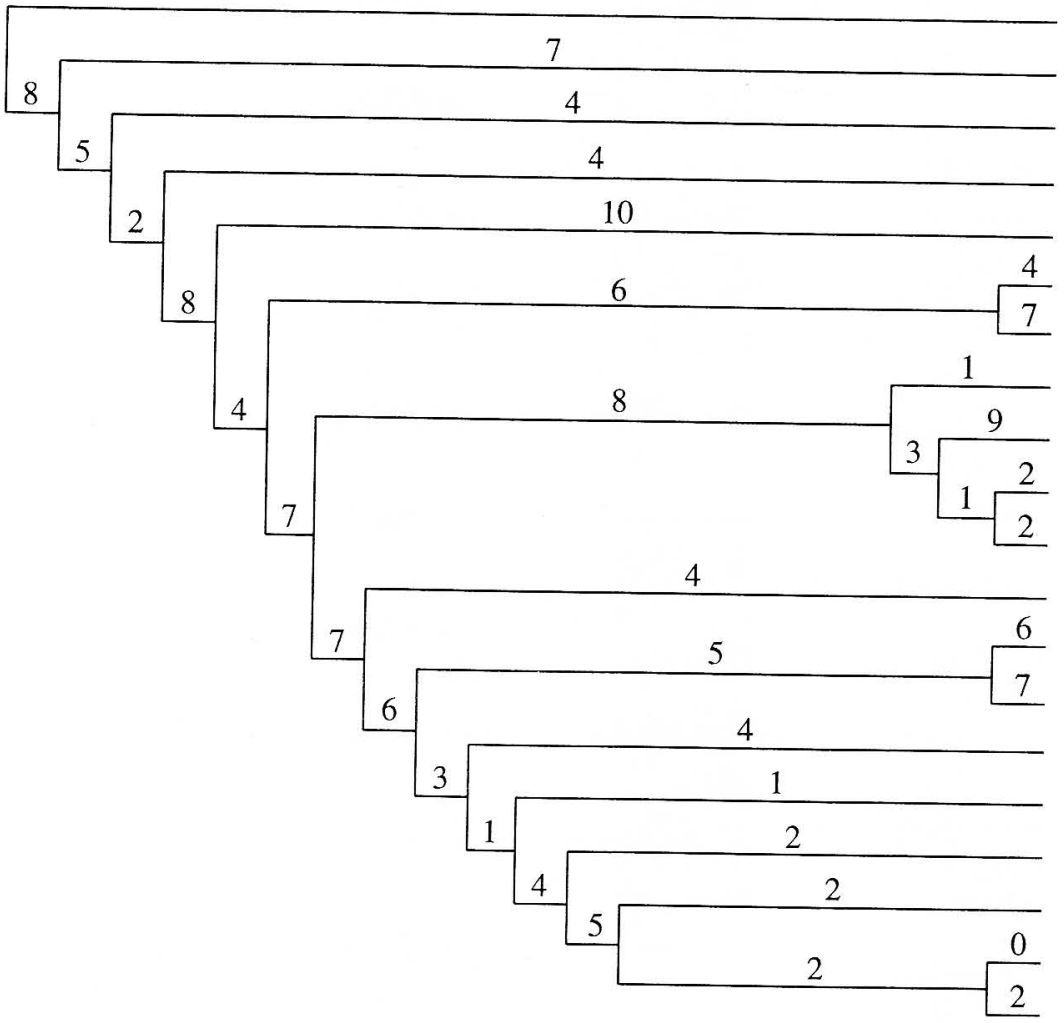

Chlorogalum

Camassia

Eremocrinum

Chlorophytum

Leucocrinum

Hesperocallis

Hosta

Hesperoyucca

Excremis

Yucca

Clistoyucca

Hesperaloë

Beschorneria

Furcraea

Agave

Agave (Litt.)

Manfreda

Prochnyanthes

Polianthes

Pseudobravoa

FIG. 1. Dos árboles igualmente más parsimoniosos obtenidos mediante el análisis con los caractercs no ordenados. Su longitud es de 163 pasos y su índice de consistencia $\mathrm{IC}=$ O. 509. Las diferencias se observan en la rama de las Yuccas. 


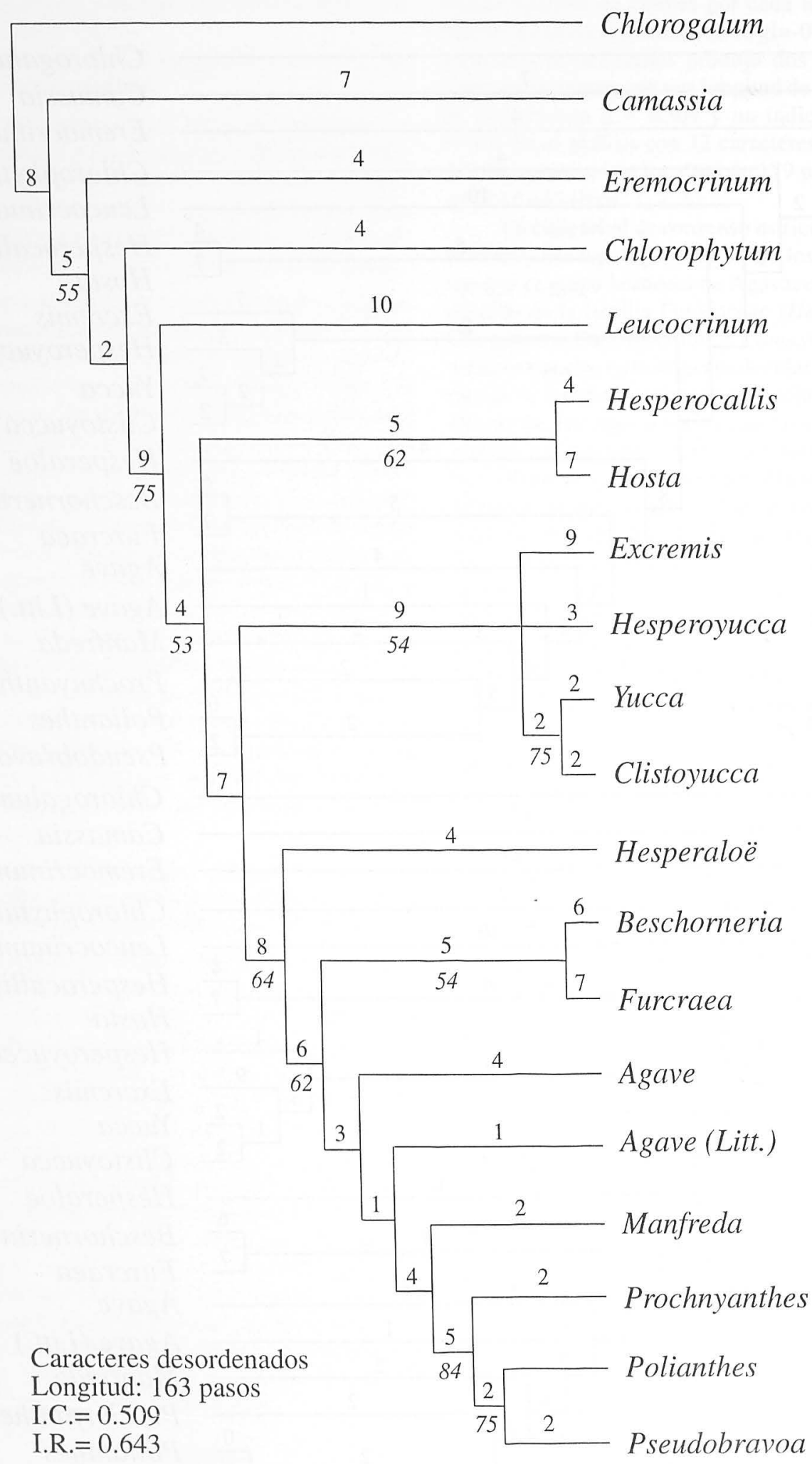




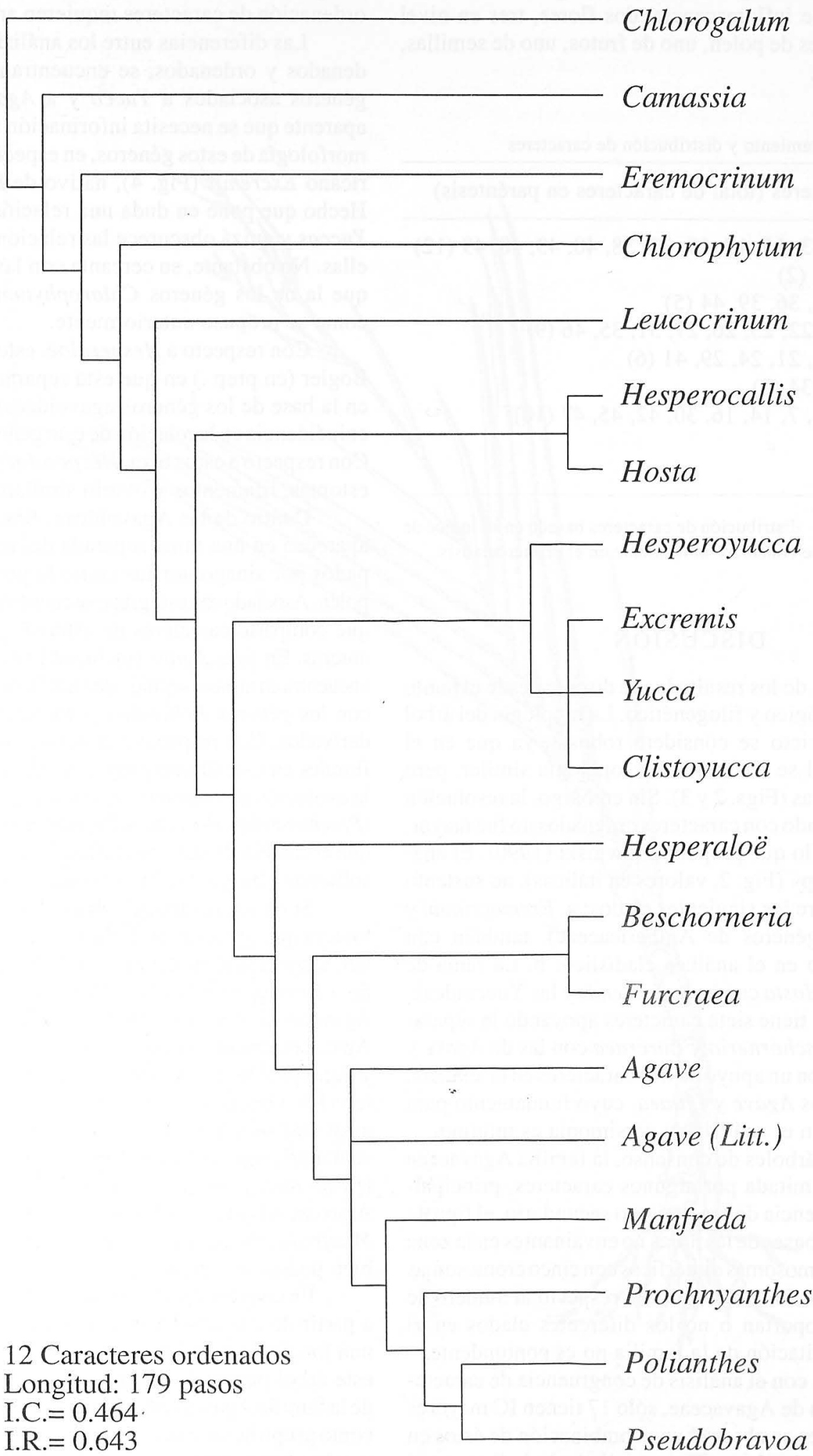

FIG. 3. Árbol de consenso estricto basado en ocho árboles igualmente más parsimoniosos producidos por el análisis con 12 caracteres ordenados. No se incluyen número de caracteres por rama ni valores de «bootstrap». 
considerados como congruentes con la filogenia, tres fueron de hábito, tres de inflorescencia, dos flores, tres en nivel embriológico, tres de polen, uno de frutos, uno de semillas, y uno citológico.

Cuadro 3. Comportamiento y distribución de caracteres

IC Caracteres (total de caracteres en paréntesis)

\begin{tabular}{ll}
\hline 1.0 & $1,6,13,15,17,19,37,38,40,43,48,49(12)$ \\
0.750 & $32,33(2)$ \\
0.667 & $18,23,36,39,44(5)$ \\
0.500 & $3,11,22,25,26,27,31,35,46(9)$ \\
0.400 & $10,12,21,24,29,41(6)$ \\
0.375 & $8,20,34(3)$ \\
0.333 & $2,4,5,7,14,16,30,42,45,47(10)$ \\
0.286 & $9(1)$ \\
0.200 & $28(\mathrm{I})$
\end{tabular}

El comportamieni. đistribución de caracteres basado en su Índice de Consistencia (IC), se consideró únicamente en el primer análisis.

\section{DISCUSIÓN}

La interpretación de los resultados se discute desde el punto de vista metodológico y filogenético. La topología del árbol de consenso estricto se considera robusta, ya que en el análisis adicional se encontró una topología similar, pero con 16 pasos extras (Figs. 2 y 3 ). Sin embargo, la resolución del árbol encontrado con caracteres ordenados no fue mayor, en contraste con lo que propone Slowinski (1990). El análisis de «bootstrap» (Fig. 2, valores en itálicas), no sustentó la separación entre los siguientes clados: a. Eremocrinum y Chlorophytum (géneros de Anthericaceae), también con poco fundamento en el análisis cladístico; b. La rama de Hesperocallis y Hosta con la de Excremis y las Yuccoideae, que en el análisis tiene siete caracteres apoyando la separación; c. La de Beschorneria y Furcraea con las de Agave y géneros afines, con un apoyo de tres caracteres en el análisis; d. Los subgéneros Agave y Littaea, cuyo fundamento para estar separados en el análisis de parsimonia es mínimo.

En los dos árboles de consenso, la familia Agavaceae se encuentra delimitada por algunos caracteres, principalmente por la presencia de crecimiento secundario, el tipo de germinación, las bases de las hojas no envainantes en la zona estéril y los 30 cromosomas dimórficos con cinco cromosomas largos y 25 cortos. Sin embargo, con respecto al número de caracteres que soportan o no los diferentes clados en el análisis, la delimitación de la familia no es contundente.

De acuerdo con el análisis de congruencia de caracteres en la filogenia de Agavaceae, sólo 17 tienen IC mayores a 0.5 , de los cuales se observó una combinación de éstos en cuanto a partes de la planta. Este resultado no indica que el resto de caracteres no sean filogenéticamente informativos, sino que la información en la matriz de datos no está completa o la formulación de árboles de los estados o la ordenación de caracteres requieren análisis más detallados.

Las diferencias entre los análisis de caracteres desordenados y ordenados, se encuentran en los clados de los géneros asociados a Yucca y a Agave (Figs. 2 y 3). Es aparente que se necesita información más detallada sobre la morfología de estos géneros, en especial del género sudamericano Excremis (Fig. 4), nativo de los Andes de Bolivia. Hecho que pone en duda una relación tan estrecha con las Yuccas y quizá obscurece las relaciones filogenéticas entre ellas. No obstante, su cercanía con las Agavaceae es mayor que la de los géneros Chlorophytum, Camassia y Hosta, como se propuso anteriormente.

Con respecto a Hesperaloë, este análisis coincide con Bogler (en prep .) en que está separado de las Yuccoideae, en la base de los géneros agavoideos; sin embargo, no hay coincidencia en la relación de este género con Hesperoyucca. Con respecto a estos taxa, Hesperaloë presenta caracteres de estomas, filamentos y ovario similares.

Dentro de las Agavoideae, Beschorneria y Furcraea aparecen en una rama separada del resto de géneros, agrupados por sinapomorfias como la presencia de tétradas en polen. Asociado con este grupo seencuentraun clado monofilético que comparte caracteres de estomas y tipo de inserción de anteras. En éste, Agave (incluyendo al subgénero Littaea) se encuentra en labase, seguido de Manfreda y luego Prochnyanthes, con los géneros Polianthes y Pseudobravoa como los más derivados. Con respecto a la evolución de ciertos caracteres florales en este último grupo, de acuerdo con los resultados, la evolución de estambres exertos a secundariamente insertos (Prochnyanthes, Polianthes, Pseudobravoa), es más parsimoniosa que el cambio de sistemas biflorales a flores secundariamente solitarias (Manfreda, Pseudobravoa) (Fig. 5).

Si de los resultados obtenidos en los cladogramas se tuviera que proponer una clasificación, aunque sea preliminar, tendría similitudes con las de Schulze (1983) excluyendo a Bravoa, con la de Dahlgren et al. (1985) y en la tribu Agaveae con la de Traub (1953). Por tanto, la familia Agavaceae estaría compuesta por dos subfamilias, Yuccoideae y Agavoideae. La primera contendría a los géneros Yucca (con los subgéneros Clistoyucca y Chaenoyucca no incluídos en el análisis), Hesperoyucca y posiblemente a Excremis. La subfamilia Agavoideae se dividiría en tres tribus, Hesperaloeae (Hesperaloë), Beschornerieae (Beschorneria, Furcraea) y Agaveae (Agave con los dos subgéneros Agave y Littaea), Manfreda, Prochnyanthes, Polianthes y Pseudobravoa, (que bien podría ser un subgénero de Polianthes).

En conclusión, se obtuvo un árbol de consenso estricto a partir de dos árboles igualmente más parsimoniosos con una longitud de 163 pasos y un IC=0.509. La topología de este árbol permitió aclarar ciertas relaciones filogenéticas de la familia Agavaceae, tales como la presencia de Excremis como grupo hermano, la relación más cercana de Hesperaloë con las Agavoideae que con las Yuccoideae, la cercanía de Agave con Manfreda y la relación más estrecha de Polianthes con Pseudobravoa que con Manfreda. 


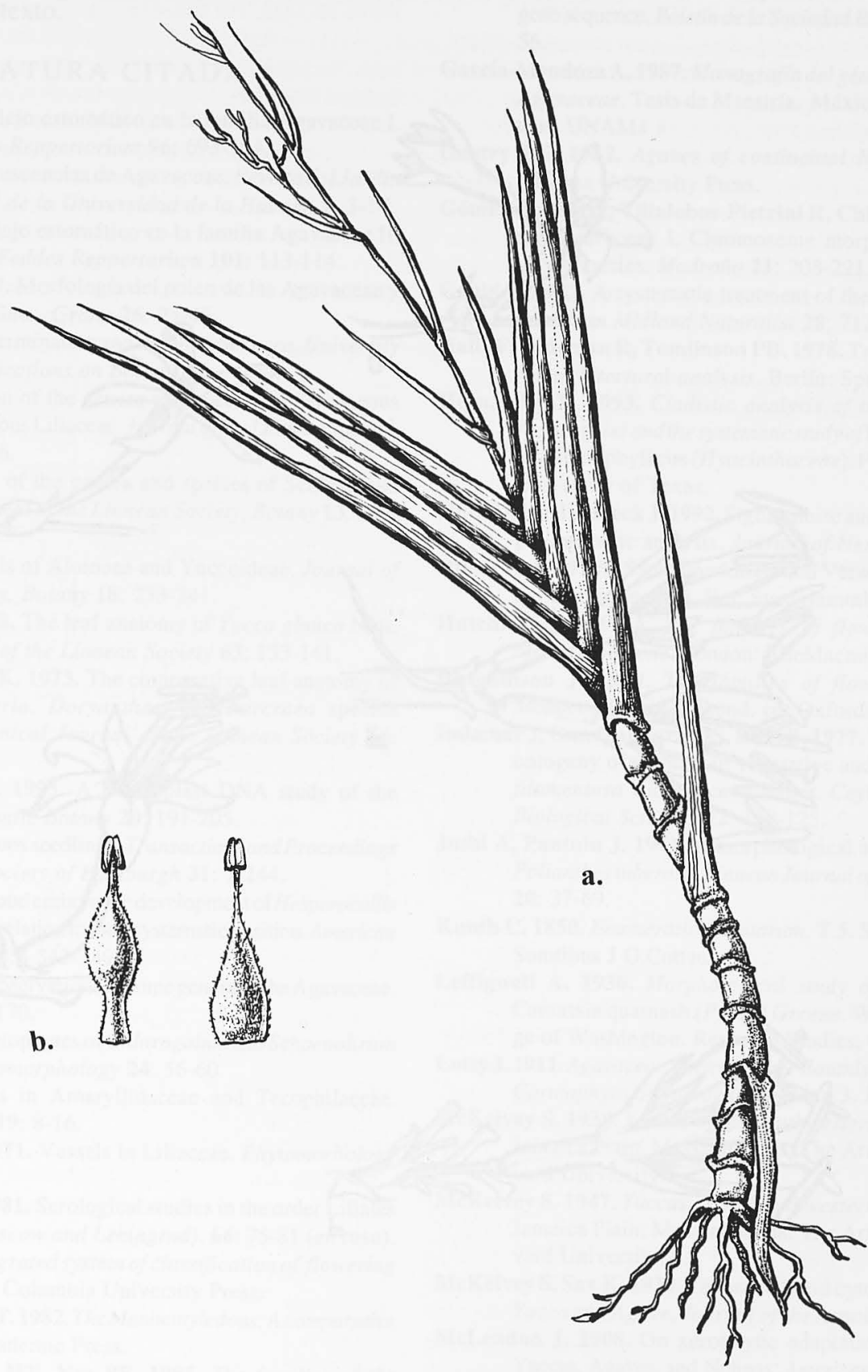



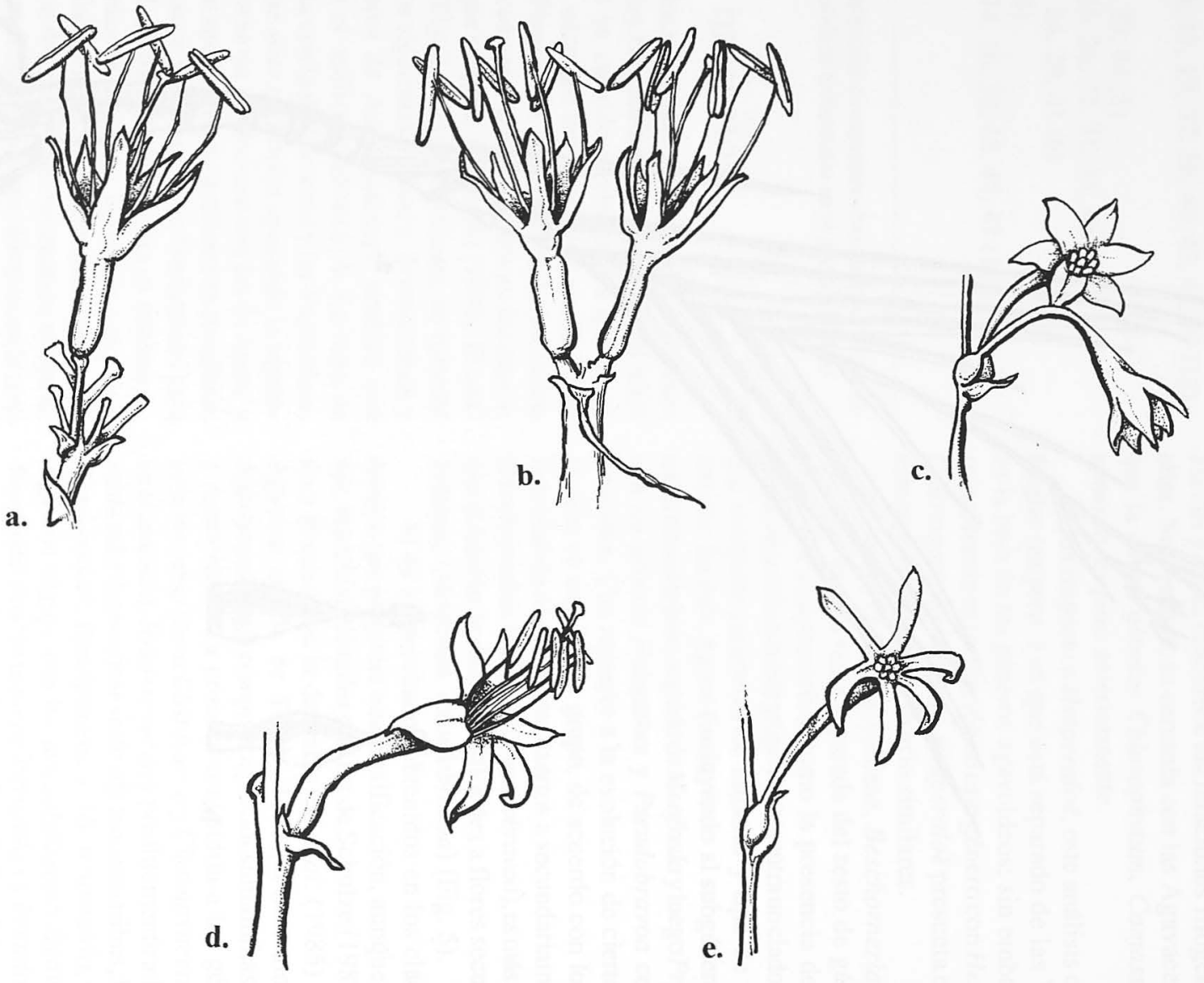


\section{AGRADECIMIENTOS}

A Patricia Dávila y Oswaldo Téllez por su apoyo técnico y moral en el desarrollo de este trabajo. A Mahinda Martínez y dos revisores anónimos por la revisión crítica del manuscrito. A Raquel Galván y Abisaí García por su esfuerzo en el desarrollo del conocimiento de la familia Agavaceae y su admirable paciencia. Al biólogo Jorge Saldívar por su ayuda en la edición final del texto.

\section{LITERATURA CITADA}

Álvarez A. 1985. El complejo estomático en la familia Agavaceae I. Desarrollo. Feddes Reppertorium 96: 693-704.

Álvarez A. 1986. Las inflorescencias de Agavaceae. Revista del Jardín Botánico Nacional de la Universidad de la Habana 7: 3-14.

Álvarez A. 1990. El complejo estomático en la familia Agavaceae II. Epidermis adulta. Feddes Reppertorium 101: 113-114.

Álvarez A, Köhler E. 1987. Morfología del polen de las Agavaceae y algunos géneros afines. Grana 26: 25-46.

Arnott H. 1962. The seed, germination and seedling of Yucca. University of California Publications on Botany 35: 7-144.

Baker JG. 1871. A revision of the genera and species of herbaceous capsular gamophyllous Liliaceae. Journal of the Linnean Society, Botany 11: 349-436.

Baker JG. 1873. Revision of the genera and species of Scilleae and Chlorogaleae. Journal of the Linnean Society, Botany 13: 209292.

Baker JG. 1881. A synopsis of Aloineae and Yuccoideae. Journal of the Linnean Society, Botany 18: 233-241.

Biunden G, Binns W. 1970. The leaf anatomy of Yucca glauca Nutt. Botanical Journal of the Linnean Society 63: 133-141.

Blunden G, Yi Y, Jewers K. 1973. The comparative leaf anatomy of Agave, Beschorneria, Doryanthes, and Furcraea species (Agavaceae). Botanical Journal of the Linnean Society 66: 157-169.

Bogler DB, Simpson BB. 1995. A chloroplast DNA study of the Agavaceae. Systematic Botany 20: 191-205.

Boyd L.1932. Monocotyledons seedlings. Transactions and Proceedings of the Botanical Society of Edinburgh 31: 1-244.

Cave M. 1948. Sporogenesis and embryo sac development of Hesperocallis and Leucocrinum in relation to their systematic position. American Journal of Botany 35: 343-349.

Cave M. 1964. Cytological observations of some genera of the Agavaceae. Madroño 17: 163-170.

Cave M. 1974. Female gametophytes of Chlorogalum and Schoenolirion (Hastingsia). Phytomorphology 24: 56-60.

Cheadle V. 1969. Vessels in Amaryllidaceae and Tecophilaceae. Phytomorphology 19: 8-16.

Cheadle V, Kosakai H. 1971. Vessels in Liliaceae. Phytomorhology 21: 320-333.

Chupov V, Kuntjavina. 1981. Serological studies in the order Liliales I. Bot Zhurnal (Moscow and Leningrad). 66: 75-81 (en ruso).

Cronquist A. 1981. An integrated system of classification of flowering plants. New York: Columbia University Press.

Dahlgren RMT, Clifford HT. 1982. The Monocotyledons: A comparative study. London: Academic Press.

Dahlgren RMT, Clifford HT, Yeo PF. 1985. The families of the monocotyledons: Structure, evolution, and taxonomy. Berlín: Springer Verlag.

Daumann E. 1970. Das Blutennektarium der Monocotyledonen unter besonderer Berucksichtigung seiner systematischen und phylogenetischen Bedeutung. Feddes Reppertorium 80: 463-590.
Duvall MR, Clegg MT, Chase M, Clark W, Kress J, Hills H, Eguiarte LE, Smith J, Gaut B, Zimmer B, Learn GH Jr. 1993. Phylogenetic hypothesis for the Monocotyledons constructed from $r b c L$ sequence data. Annals of the Missouri Botanical Garden 80: 607-619.

Eguiarte LE, Duvall MR, Learn GH Jr, Clegg MT. 1994. The systematic status of the Agavaceae and Nolinaceae and related Asparagales in the Monocotyledons: An analysis based on the $r b c L$ gene sequence. Boletín de la Sociedad Botánica de México 54: 3556.

García-Mendoza A. 1987. Monografía del género Beschorneria Kunth. Agavaceae. Tesis de Maestría. México DF: Facultad de Ciencias, UNAM.

Gentry HS. 1982. Agaves of continental North America. Tucson: Arizona University Press.

Gómez-Pompa A, Villalobos-Pietrini R, Chimal A. 1971. Studies in the Agavaceae I. Chromosome morphology and number of seven species. Madroño 21: 208-221.

Gould F. 1942. A systematic treatment of the genus Camassia Lindl. American Midland Naturalist 28: 712-742.

Hallè F, Oldeman R, Tomlinson PB. 1978. Tropical trees and forests. An architectural analysis. Berlín: Springer Verlag.

Hernández L. 1993. Cladistic analysis of the American genera of Asparagales and the systematic study of Beaucarnea (Nolinaceae) and Hemiphylacus (Hyacinthaceae). PhD thesis. Austin, USA: University of Texas.

Hillis D, Huelsenbeck J. 1992. Signal, noise and reliability in molecular phylogenetic analysis. Journal of Heredity 83: 189-195.

Huber H. 1969. Die Samenmerkmale und Verwandtschaftsverhaltnisse der Liliflorae. Mitt. Bot. Staatssamml. München. 8: 219-538.

Hutchinson J. 1934. The families of flowering plants. Vol. II. Monocotyledons. London: TheMacmillan Co.

Hutchinson J. 1959. The families of flowering plants. Vol. II. Monocotyledons. Second. ed. Oxford: Clarendon Press.

Indamar J, Gandgadhara MS, Bhat B. 1977. Epidermal structure and ontogeny of stomata in vegetative and floral organs of Yucca filamentosa var. concava Baker. Ceylon Journal of Science, Biological Sciences 12: 119-125.

Joshi A, Pantulu J. 1941. A morphological and cytological study of Polianthes tuberosa. Linnean Journal of India Botanical Society 20: 37-69.

Kunth C. 1850. Enumeratio Plantarum. T.5. Stutgardiae et Tubingae, Sumtibus J G.Cottae.

Leffigwell A. 1930. Morphological study of bulb and flowers of Camassia quamash (Pursh) Greene. Washington: State College of Washington. Research Studies, Contribution 28.

Lotsy J. 1911. Agavaceae Vorträge über Botanische Stammesgeschichte. Cormophyta Siphonogamia, Band 3. Jena: G Fischer.

McKelvey S. 1938. Yuccas of the southwestern United States. Part 1. Jamaica Plain, Massachusetts: The Arnold Arboretum of Harvard University.

McKelvey S. 1947. Yuccas of the Southwestern United States. Part II. Jamaica Plain, Massachusetts: The Arnold Arboretum of Harvard University.

McKelvey S, Sax K. 1933. Taxonomic and cytological relationships of Yucca and Agave. Journal of the Arnold Arboretum 14: 76-81.

McLendon J. 1908. On xerophytic adaptations of leaf structure in Yuccas, Agaves, and Nolinas. American Naturalist 42: 308-316.

Mikevich M, Weeler S. 1990. Evolutionary character analysis: tracing character change on cladogram. Cladistics 6: 137-170.

Ojeda L. 1988. Palinología de Agavaceae, una contribución sistemática. Tesis de Maestría. México DF: Facultad de Ciencias, UNAM.

Ojeda $\mathbb{L}$, Ludlow-Wiechers B, Orellana R. 1984. Palinología de la 
familia Agavaceae para la península de Yucatán. Biótica 9: 379-398.

Patterson C. 1988. Homology in classical and molecular biology. Molecular Biology and Evolution 5: 603-625.

Pax F, Hoffman K. 1930. Amaryllidaceae. En: Engler A, Prantl K. (Eds.). Die Natürlichen Pflanzenfamilien. Leipzzig: Engelmann.

Rose JN. 1899. Studies of Mexican and Central American plants No. 2. A proposed rearrangement of the suborder Agaveae. Contributions of the United States National Herbarium 5: 150-157.

Rose JN. 1903. Studies of Mexican and Central American plants. Amaryllidaceae. Contributions of the United States National Herbarium 8: 8-23.

Satô D. 1935. Analysis of the karyotypes in Yucca, Agave and related genera with special reference to the phylogenetic significance. Japanese Journal of Genetics 11: 273278.

Sattler R. 1994. Homology, homeosis, and process morphology in plants. En: Hall K. (Ed.). Homology. San Diego: Academic Press.

Schulze W. 1983. Beitrage zur Taxonomie der Lilifloren. XII. Der umfang der Agavaceae. Wissenschaft Zentralicher FriederichSchiller-Universität Jena, Mathematik-Natumwissenschaft 32: 965-979.

Sen S. 1975. Cytotaxonomy of Liliales. Feddes Reppertorium 86: 255-305.

Slowinski J. 1993. «Unordered» versus «ordered» characters. Systematic
Biology 42: 155-165.

Swofford D. 1990. Phylogenetic Analysis Using Parsimony. Version 3.0. Champaign, Illinois: Illinois Natural History Survey.

Takhtajan AL. 1980. Outline of the classification of flowering plants (Magnoliophyta). Botanical Review 46: 225-359.

Tomlinson B. 1984. Vegetative morphology -some enigmas in relation to plant systematics-. En: Heywood V, Moore D. (Eds.). Current concepts in plant taxonomy. London: Academic Press.

Traub H. 1953. The tribes and genera of the Agavaceae. Plant Life 9: 134-137.

Troll W. 1964. Die inflorezenzen. Stuttgart: Fischer Verlag.

Verhoek WS.1975. A study of the tribe Poliantheae (including Manfreda) and revisions of Manfreda and Prochnyanthes (Agavaceae). PhD thesis. Ithaca, New York: Cornell University.

Webber JM. 1953. Yuccas of the Southwest. Agricultural Research Service U.S. Department of Agriculture. Agriculture Monographs 17.

Whitaker JW.1934. Chromosome constitution in certain Monocotyledons. Journal of the Arnold Arboretum 31: 283-289.

Wunderlich R. 1950. Die Agavaceae Hutchinsons im lichte ihrer Embriologie, ihres Gynözeum-, Staubblatt und Blattbaues. En: Geitler L. (Ed.). Österreichische Botanische Zeitschrift. Viena: Springer Verlag. 